\title{
Gauss Law Constraints in Chern-Simons Theory From BRST Quantization
}

\author{
M. Chaichian ${ }^{a, b}$, W. F. Chen ${ }^{b \dagger}$ and Z.Y. Zhu ${ }^{c, d}$ \\ ${ }^{a}$ High Energy Physics Laboratory, Department of Physics \\ ${ }^{b}$ Research Institute for High Energy Physics, University of Helsinki \\ P.O. Box 9 (Siltavuorenpenger 20 C), FIN-00014 Helsinki, Finland \\ ${ }^{c}$ CCAST(World Laboratory), P.O. Box 8730, Beijing 100080, China \\ ${ }^{d}$ Institute of Theoretical Physics, Academia Sinica, P. O. Box 2735, Beijing, 100080, China
}

\begin{abstract}
The physical state condition in the BRST quantization of Chern-Simons field theory is used to derive Gauss law constraints in the presence of Wilson loops, which play an important role in explicitly establishing the connection of Chern-Simons field theory with 2-dimensional conformal field theory.
\end{abstract}

When we discuss knot invariants in terms of Chern-Simons theory 106 and the relationship between Chern-Simons field theory and conformal field theory relation is Gauss law constraint in presence of Wilson line, which was first given in ref. [1]. This relation plays an important role in proving that states of Chern-Simons theory satisfy the Knizhnik-Zamolodchikov equation 913 . In this letter we intend to derive the Gauss law constraints from BRST quantization of Chern-Simons field theory. We think this investigation is significant since in some sense BRST quantization formulation is defined better than a formal manipulation without gauge fixing 4 . We will show that when Wilson lines exist the physical state condition in BRST quantization will lead to Gauss law constraints

\footnotetext{
${ }^{\dagger}$ ICSC-World Laboratory, Switzerland
} 
with source terms just as those given in ref. [1]. The procedure we will adopt is similar to the one in ref. [15], where the equivalence between Dirac's first-class constraints and BRST treatment for Yang-Mills theory is formally proved.

Let us first write down the BRST quantization of Chern-Simons theory. The action of Chern-Simons field theory takes the following form

$$
S_{\mathrm{CS}}=\frac{k}{4 \pi} \int_{M^{3}} \operatorname{Tr}\left[A \wedge d A+\frac{2}{3} A \wedge A \wedge A\right],
$$

where $A=A_{\mu} d x^{\mu}=A_{\mu}^{a} T^{a} d x^{\mu}$ with $T^{a}$ being the generators in some representation of gauge group $G$. The parameter $k$ must be chosen to be an integer in order to make the theory gauge invariant under large gauge transformations. Without loss of generality, we choose $G=S U(N)$ and the normalization $\operatorname{Tr}\left(T^{a} T^{b}\right)=\frac{1}{2}$. Choosing the Lorentz gauge $\partial^{\mu} A_{\mu}^{a}=0$ and performing BRST gauge fixing, we obtain the following effective action

$$
\begin{aligned}
S_{\mathrm{eff}}= & \int d^{3} x \mathcal{L}_{\mathrm{eff}}=S_{\mathrm{CS}}+\int \operatorname{Tr} \delta\left[\bar{c}\left(\partial_{\mu} A^{\mu}+B\right)\right] \\
= & \int d^{3} x\left\{\frac{k}{16 \pi} \epsilon^{\mu \nu \rho}\left[A_{\mu}^{a}\left(\partial_{\nu} A_{\rho}^{a}-\partial_{\rho} A_{\nu}^{a}\right)+i \frac{2}{3} f^{a b c} A_{\mu}^{a} A_{\nu}^{b} A_{\rho}^{c}\right]\right. \\
& \left.-\frac{i k}{8 \pi} A^{\mu a} \partial_{\mu} B^{a}+\frac{i k}{8 \pi}\left(B^{a}\right)^{2}-\frac{1}{2} \partial_{\mu} \bar{c}^{a} D^{\mu} c^{a}\right\},
\end{aligned}
$$

where $B=B^{a} T^{a}$ is the auxiliary field and $A_{\mu}=A_{\mu}^{a} T^{a}, c=c^{a} T^{a}, \bar{c}=\bar{c}^{a} T^{a}$. The BRST transformations of the fields are as follows

$$
\begin{aligned}
& \delta A_{\mu}^{a}=D_{\mu} c^{a}, \delta B^{a}=0, \\
& \delta c^{a}=-\frac{1}{2} f^{a b c} c^{b} c^{c}, \delta \bar{c}^{a}=\frac{i k}{4 \pi} B^{a} .
\end{aligned}
$$

These transformations are nilpotent, i.e., $\delta^{2}=0$. Now, obviously the classical configuration space is enlarged by the introduction of new fields - ghost fields $c^{a}$, anti-ghost fields $\bar{c}^{a}$ and multiplier fields $B^{a}$. The canonically conjugate momenta can be well defined by $\Pi_{\Phi}=\frac{\partial \mathcal{L}}{\partial \dot{\Phi}}$, with $\Phi=\left\{A_{1}, B, c, \bar{c}\right\}$ :

$$
\begin{aligned}
\Pi_{A_{1}}^{a} & =\frac{k}{8 \pi} A_{2}^{a}, \Pi_{B}^{a}=-i \frac{k}{8 \pi} A_{0}^{a}, \\
\Pi_{\bar{c}}^{a} & =-\frac{1}{2} D^{0} c^{a}, \Pi_{c}^{a}=\frac{1}{2} \dot{\bar{c}}^{a} .
\end{aligned}
$$


These fields and their canonically conjugate momenta satisfy the Poisson brackets (for bosonic fields) or anti-brackets (for fermionic fields):

$$
\begin{aligned}
\left\{\Pi_{\Phi}^{i}(\mathbf{x}, t), \Phi_{j}(\mathbf{y}, t)\right\}_{ \pm \mathrm{PB}} & =-i \delta_{j}^{i} \delta^{(2)}(\mathbf{x}-\mathbf{y}) \\
\left\{\Pi_{\Phi}^{i}(\mathbf{x}, t), \Pi_{\Phi}^{j}(\mathbf{y}, t)\right\}_{ \pm \mathrm{PB}} & =\left\{\Phi^{i}(\mathbf{x}, t), \Phi^{j}(\mathbf{y}, t)\right\}_{ \pm \mathrm{PB}}=0 .
\end{aligned}
$$

The BRST charge can be obtained by the Noether theorem

$$
\begin{aligned}
Q & =\int d^{2} x\left[\frac{k}{8 \pi} \epsilon^{i j} D_{i} c^{a} A_{j}^{a}-\frac{1}{4} f^{a b c} \dot{\bar{c}}^{a} c^{b} c^{c}-\frac{i k}{8 \pi} B^{a} D^{0} c^{a}\right] \\
& =\int d^{2} x\left[-\frac{k}{8 \pi} c^{a} F_{12}^{a}-\frac{1}{2} f^{a b c} \Pi_{c}^{a} c^{b} c^{c}+\frac{i k}{4 \pi} B^{a} \Pi_{\bar{c}}^{a}\right]
\end{aligned}
$$

It is easy to show that

$$
\delta \Phi=\{Q, \Phi\}_{ \pm \mathrm{PB}},\{Q, Q\}_{ \pm \mathrm{PB}}=Q^{2}=0
$$

When we perform quantization, the classical observables are replaced by operators, and (anti-) Poisson brackets by (anti-) commutative Lie brackets. With the present polarization choice, the Hilbert space are composed of square integrable functionals of $\Phi$. The quantum BRST charge operator $\hat{Q}$ is nilpotent

$$
\frac{1}{2}\{\hat{Q}, \hat{Q}\}=\hat{Q}^{2}=0
$$

where a hat " "» denotes an operator. It is well known that the state space here possesses indefinite metric. According to the general principle of BRST quantization, physical states satisfy the so-called "BRST-closed" condition

$$
\hat{Q} \mid \text { phys }\rangle=0 \text {. }
$$

Notice that above condition (9) determines a physical state up to "BRST-exact states", i.e.

$$
\mid \text { phys }\rangle \sim \mid \text { phys }\rangle+|\chi\rangle,|\chi\rangle=\hat{Q} \mid \text { any states }\rangle
$$

Obviously these states $|\chi\rangle$ are normal to all physical states including themselves,

$$
\langle\chi| \text { phys }\rangle=\left\langle\chi_{1} \mid \chi_{2}\right\rangle=0
$$


Thus they are zero norm states and make no contribution to the physical observables. Now we define the physical operator $\hat{\Phi}$ to be an operator that generates a physical state from vacuum. It is easy to show that the physical operator $\hat{\Phi}$ must satisfy the condition

$$
[\hat{\Phi}, \hat{Q}]_{ \pm}=f[\hat{\Phi}] \hat{Q}
$$

due to Eq.(9). Furthermore, the operators can be divided into two classes. According to ref. [15], we call them as the A-type and the B-type. An A-type operator transforms a physical state into another one

$$
\hat{A} \mid \text { phys }\rangle=\mid \text { phys }\rangle^{\prime} \text {. }
$$

A B-type operator transforms a physical state into a BRST exact state and has the form

$$
\hat{B}=[*, \hat{Q}]_{ \pm}
$$

where $*$ represents some operator. Eq.(14) implies that a B-type operator can be regarded as the generator of a kind of gauge transformation since it does not affect physical observables. The (anti-)commutators of B-type operators with an arbitrary physical operator $\hat{\Phi}$ have the form

$$
\left[\hat{B}_{i}, \hat{\Phi}\right]_{ \pm}=g[\hat{\Phi}]_{i j} \hat{B}_{j}
$$

which means that B-type operators form an ideal in the operator algebra 15

$$
\begin{aligned}
& {[\hat{A}, \hat{A}]_{ \pm} \subset\{\hat{A} \& \hat{B}\}} \\
& {[\hat{A}, \hat{B}]_{ \pm} \subset\{\hat{B}\},[\hat{B}, \hat{B}]_{ \pm} \subset\{\hat{B}\}}
\end{aligned}
$$

The product of an arbitrary operator $\hat{K}$ (physical or nonphysical) with a B-type operator can also be regarded as the generator of gauge transformations due to the fact that

$$
\left[(\hat{K} \hat{B})_{i}, \hat{\Phi}\right]_{ \pm}=h[\hat{\Phi}]_{i j}(\hat{K} \hat{B})_{j}
$$

In addition, $\hat{K} \hat{B}$ operators also form a closed algebra 


$$
\left.\left[(\hat{K} \hat{B})_{i}, \hat{K} \hat{B}\right)_{j}\right]_{ \pm}=U_{i j}^{k}(\hat{K} \hat{B})_{k}
$$

Notice that the $\hat{K} \hat{B}$ operator transforms a physical state out of the genuine physical state space 15 . We can see in the following that the properties of $\hat{B}$ or $\hat{K} \hat{B}$ operators play a crucial role in our derivation.

From the BRST charge given in Eq.(6) we can show that

$$
\begin{aligned}
\hat{B}_{1}^{a} & \equiv\left[\hat{Q}, \hat{\Pi}_{c}^{a}\right]=-\frac{k}{8 \pi} \hat{F}_{12}^{a}-\frac{1}{2} f^{a b c} \hat{\Pi}_{c}^{b} \hat{c}^{c}, \\
\hat{B}_{2}^{a} & \equiv\left[\hat{Q}, \hat{\Pi}_{B}^{a}\right]=\frac{k}{4 \pi} \hat{\Pi}_{c}^{a}, \\
\hat{B}_{3}^{a} & \equiv\left[\hat{Q}, \partial^{\mu} \hat{A}_{\mu}^{a}\right]=M_{a b} \hat{c}^{b},
\end{aligned}
$$

where $M_{a b}=\frac{k}{8 \pi}\left[\hat{F}_{12}^{a}, \partial^{\mu} \hat{A}_{\mu}^{b}\right]$. Furthermore, we know that the matrix $\left(M_{a b}\right)$ is non-singular from the fact that $\hat{F}_{12}$ and $\partial^{\mu} \hat{A}_{\mu}$ constitute a pair of second-class constraints 12,12 . Note that in deriving the Eqs.(19) we have used the $B$-field equation of motion (on-shell condition). The non-singularity of $M$ ensures that ghost field operators can be written as $\hat{c}^{a}=\left(M^{-1}\right)^{a b} \hat{B}_{3}^{b}$ and belong to the B-type. Hence they are indeed the generators of gauge transformations.

From Eqs.(6), (19) and the above arguments, one can see that the three terms composed of BRST charge $\hat{Q}$ are all gauge transformation generators. However the second and third terms are $\hat{B}$ - or $\hat{K} \hat{B}$-type operators. Thus when $\hat{Q}$ acts on physical states, the second and the third terms transform the physical state to non-physical state. Since there exists no coupling between the nonphysical gauge transformation generators $\hat{c}^{a}$ and the physical ones, after the action of BRST charge, the transformed state $\mid>$ can be written as

$$
|\rangle=\mid \text { non-phys }\rangle \oplus \mid \text { phys }\rangle \text {. }
$$

So the physical state condition $\hat{Q} \mid$ phys $\rangle=0$ reduces to

$$
\hat{F}_{12}^{a}|p h y s\rangle=0
$$

when no Wilson loop exists. Now we turn to the case in the presence of Wilson loops. Let us take the manifold $M=\Sigma \times R$ as in ref. [四], where $R$ is the time variable space and $\Sigma$ 
is the spatial surface. The physical state at some time $t$ in the presence of a Wilson loop can be represented by a punctured surface $\Sigma$, the puncture points being produced by the intersections of the surface $\Sigma$ with the links where the Wilson loop operators are defined. This has been given in ref. [16]

$$
\mid \text { phys }\rangle=\Pi_{n=1}^{N} \exp \left[i \int_{P_{n}\left(\Gamma_{n}\right)}^{Q_{n}} \sum_{i=1,2} \hat{A}_{i}^{(n)}(\mathbf{x}) d x^{i}\right]|0\rangle,
$$

where $n$ denotes the $n$th component of links, $\Gamma_{n}$ is the projection on $\Sigma$ of links located in the three dimensional space-time region less than time $t$ and $P_{n}, Q_{n}$ are the endpoints of $\Gamma_{n}$. In the polarization chosen above, the state functional can be written down explicitly in a path integral form 16

$$
\begin{aligned}
& \left.\Psi_{\text {phys }}[\Phi]=\langle\hat{\Phi}| \text { phys }\right\rangle \\
& =\left(\int \mathcal { D } \Xi ^ { \prime } \left\{\exp \left[i \int_{-\infty}^{t} d t^{\prime} \int_{\Sigma} d^{2} x \mathcal{L}_{\text {eff }}-\frac{k}{2 \pi} \int_{\Sigma} d^{2} x \sum_{i=1}^{2} A_{i}^{\prime a} A_{i}^{\prime a}\right]\right.\right. \\
& \left.\left.\times \prod_{n=1}^{N} \exp \left[i \int_{P_{n}}^{Q_{n}} \sum_{i=1}^{2} A_{i}^{\prime(n)}(x) d x^{i}\right] \Psi_{0}\right\}\right)[\Phi] \\
& \Phi=\left(A_{1}, B, c, \bar{c}\right), \quad \Xi=\left(A_{\mu}, B, c, \bar{c}\right), \mu=0,1,2 .
\end{aligned}
$$

where $\Psi_{0}$ is the vacuum state functional at time $t=-\infty$ and it is determined by Eq.(21). Eq.(23) is in fact the gauge-fixed version of the state functional given in ref. [10]. Therefore, we have that

$$
\begin{aligned}
& \hat{Q} \mid \text { phys }\rangle=\left\{\int d^{2} x\left[\frac{k}{8 \pi} \epsilon^{i j} D_{i} \hat{c}^{a} A_{j}^{a}-\frac{1}{4} f a b \hat{c} \hat{\bar{c}}^{a} \hat{c}^{b} \hat{c}^{c}-\frac{i k}{8 \pi} \hat{B}^{a} D^{0} \hat{c}^{a}\right]\right. \\
& \left.\times \Pi_{n=1}^{N} \exp \left[i \int_{P_{n}\left(\Gamma_{n}\right)}^{Q_{n}} \sum_{i=1,2} \hat{A}_{i}^{(n)}\left(x_{1}, x_{2}\right)\right]\right\}|0\rangle \\
& =\left\{\int d ^ { 2 } x \left[-\hat{c}^{a}\left[\frac{k}{8 \pi} \hat{F}_{12}^{a}-\sum_{n=1}^{N} T_{(n)}^{a}\left(\delta^{(2)}\left(\mathbf{x}-\mathbf{x}_{P_{n}}\right)-\delta^{(2)}\left(\mathbf{x}-\mathbf{x}_{Q_{n}}\right)\right]\right.\right.\right. \\
& \left.\left.\left.+\frac{k}{4 \pi} \hat{B}^{a} \hat{\Pi}_{c}^{a}-\frac{1}{2} f^{a b c} \hat{\Pi}_{c}^{a} \hat{c}^{b} \hat{c}^{c}\right]\right\} \mid \text { phys }\right\rangle \\
& =\left\{\int d^{2} x\left[-\hat{c}^{a}\left[\frac{k}{8 \pi} \hat{F}_{12}^{a}-\sum_{n=1}^{N} T_{(n)}^{a}\left(\delta^{(2)}\left(\mathbf{x}-\mathbf{x}_{P_{n}}\right)-\delta^{(2)}\left(\mathbf{x}-\mathbf{x}_{Q_{n}}\right)\right]\right]\right\} \mid \text { phys }\right\rangle \\
& \oplus\left|\chi_{1}\right\rangle>\oplus\left|\chi_{2}\right\rangle>=0,
\end{aligned}
$$

where Eq.(6) and the following operator equations have been used: 


$$
\begin{aligned}
& \hat{A}_{2}^{a}=\frac{8 \pi}{k} \hat{\Pi}_{A_{1}}^{a}=-\frac{8 i \pi}{k} \frac{\delta}{\delta \hat{A}_{1}^{a}}, \\
& {\left[\partial_{P_{n}}^{\mathbf{x}} \frac{\delta}{\delta \hat{A}_{1}^{a}\left(\mathbf{x}_{P_{n}}\right)}, \exp \left[i \int_{P_{n}\left(\Gamma_{n}\right)}^{Q_{n}} \sum_{i=1,2} \hat{A}_{i}^{(n)}(\mathbf{x}) d x^{i}\right]\right]=-i T_{(n)}^{a} \delta^{(2)}\left(\mathbf{x}-\mathbf{x}_{P_{n}}\right)} \\
& \times \exp \left[i \int_{P_{n}\left(\Gamma_{n}\right)}^{Q_{n}} \sum_{i=1,2} \hat{A}_{i}^{(n)}(\mathbf{x}) d x^{i}\right], \\
& {\left[\partial_{Q_{n}}^{\mathbf{x}} \frac{\delta}{\delta \hat{A}_{1}^{a}\left(\mathbf{x}_{Q_{n}}\right)}, \exp \left[i \int_{P_{n}\left(\Gamma_{n}\right)}^{Q_{n}} \sum_{i=1,2} \hat{A}_{i}^{(n)}(\mathbf{x}) d x^{i}\right]\right]=i T_{(n)}^{a} \delta^{(2)}\left(\mathbf{x}-\mathbf{x}_{Q_{n}}\right)} \\
& \times \exp \left[i \int_{P_{n}\left(\Gamma_{n}\right)}^{Q_{n}} \sum_{i=1,2} \hat{A}_{i}^{(n)}(\mathbf{x}) d x^{i}\right] .
\end{aligned}
$$

Thus, the physical state condition $\hat{Q} \mid$ phys $\rangle=0$, can be reduced to the form

$$
\left.\left[\frac{k}{8 \pi} \hat{F}_{12}^{a}-\sum_{n=1}^{N} T_{(n)}^{a}\left(\delta^{(2)}\left(\mathbf{x}-\mathbf{x}_{P_{n}}\right)-\delta^{(2)}\left(\mathbf{x}-\mathbf{x}_{Q_{n}}\right)\right)\right] \mid \text { phys }\right\rangle=0 .
$$

These are exactly the Gauss law constraints given by Witten in the case that Wilson loop operators are present.

Acknowledgment: WFC is grateful to the World Laboratory, Switzerland, for financial support. 


\section{REFERENCES}

[1] E. Witten, Comm. Math. Phys. 121 (1989) 351.

[2] E. Guadagnini, Int. J. Mod. Phys. A7 (1992) 87.

[3] J.F.W.H. van de Wetering, Nucl. Phys. B379 (1992) 172.

[4] L. Rozansky and H. Saleur, Nucl. Phys. B376 (1992) 461.

[5] J.H. Horne, Nucl. Phys. B334 (1990) 669.

[6] R.K. Kaul and T.R. Govindarajan, Nucl. Phys. B380 (1992) 293.

[7] L. Alvarez-Gaumé, C. Gomez and G. Sierra, Topics in Conformal Field Theory in Physics and Mathematics of String, 16-184, ed. by L. Brink, 1990.

[8] G. Moore and N. Seiberg, Phys. Lett. B220 (1989) 422.

[9] M. Bos and V.P. Nair, Int. J. Mod. Phys. A5 (1990) 959.

[10] J.M.F. Labastida and A.V. Ramallo, Phys. Lett. B228 (1989) 214; B227 (1989) 92;

J.M.F. Labastida, P.M. Platas and A.V. Ramallo, Nucl. Phys. B348 (1991) 651.

[11] S. Elitzur, G. Moore, A. Schwimmer and N. Seiberg, Nucl. Phys. B326 (1989) 108.

[12] P. Ginsparg, Applied Conformal Field Theory in Field, String and Critical Phenomena, 1988, ed. by E. Brezin and J. Zinn-Justin.

[13] V.I. Knizhnik and A. Zamolodchikov, Nucl. Phys. B247 (1984) 83.

D. Gepner and E. Witten, Nucl. Phys. B278 (1986) 493.

[14] T. Kugo and I. Ojima, Supp. Prog. Theor. Phys. 66 (1979) 1.

[15] R. Marnelius, Phys. Lett. B99 (1981) 467.

[16] E. Guadagnini, M. Martellini and M. Mintchev, Nucl. Phys. B336 (1990) 581.

[17] P.A.M. Dirac, Lectures on Quantum Mechanics, Yeshiva University, New York, 1964. 
[18] M. Henneaux and C. Teitelboim, Quantization of Gauge Systems, Princeton University Press, Princeton, 1992. 